PRENATAL AND PERINAIIAL DISORDERS

\title{
BIRIH COMPLICATIONS AND RISK OF CEREBRAL PALSY
}

Statisticians at the National Institutes of Health (NINCDS), Bethesda, MD have compared the follow-up neurologic status of full-term infants whose birth was complicated but who were free of signs of hypoxic-ischemic encephalopathy (HIE) in the nursery period with asymptomatic infants whose births were uncomplicated . The rate of $\mathrm{CP}$ was $2.3 / 1000$ in asymptomatic infants with one or more birth complications and 2.4/1000 among asymptomatic infants whose births were uncomplicated. A total of 41,012 patients was evaluated at 7 years of age in the National Collaborative Perinatal Project. The full-term infant whose birth was complicated but who was free from abnormal signs of HIE in the newborn period was not at increased risk of CP. The neonatal signs judged indicative of HIE were (1) decreased activity after the first day of life, (2) need for incubator care for three or more days, (3) feeding problems, (4) poor suck, (5) respiratory difficulty, and/or (6) neonatal seizure. (Nelson KB, Ellenberg JH. The asymptomatic newborn and risk of cerebral palsy. AJDC $1987 ; 141$ : 1333-1335).

COwBNr. Term infants whose brains have been injured during delivery show signs of damage in the neonatal period. Children with sustained neurologic abnormality in the newborn period are at a higher risk of $\mathrm{CP}$ than those with transient abnormalities. The risk increases directly with the number of abnormal signs. Neonatal seizures are the most reliable evidence of intrapartum asphyxia. The Apgar score is not the best indicator. In the NCPP, the proportion of CP attributable to asphyxia - as defined by a 5 -min Apgar, score score of <6-was about $25 \%$. Most children with CP do not have low Apgars at birth. Only about $12 \%$ of surviving children with Apgar scores of 0-3 developed CP (Nelson K, Ellenberg J. Pediatrics $1981 ; 68 ; 36)$. For babies over $2500 \mathrm{gm}$, irrespective of birth complication, most achieved 5-min Apgar scores of 7 or higher, and the risk of CP was not significantly higher than in children whose births were uncomplicated. The risk of CP attending low Apgars was conditioned on low birth weight, and $25 \%$ of $\mathrm{CP}$ cases are attributable to premature delivery (Freeman JM, Editor, Prenatal and perinatal factors associated with brain disorders. NIH Publication 1985; No 85-1149).

\section{CEREBETLAR ATROPHY AND AUTISH}

Magnetic resonance imaging scans (MRI) were obtained for 14 autistic patients, aged 4 to 19 yrs, diagnosed by DSM-III criteria at the Univ of Iowa Hospitals, Iowa City, and were compared to a control group. In coronal scans, the cerebellum of autistic patients was smaller and the IVth ventricles larger whereas axial scans showed no differences from controls. The authors refer to previous reports of postmortem evidence of cerebellar pathology in autism (Bauman $M$, Kemper TL. Neurology $1985 ; 35: 866)$, and CT scans showing cerebellar atrophy (Damasio $\mathrm{H}$ et al. Arch Neurol 1980; 37: 504). The significance of 
cerebellar involvement in behavioral disorders is unknown. (Gaffney GR et al. Cerbellar structure in autism. AJDE 1987; 141:1330-1332).

Correvr. The recognition of an organically based dysfunction of the brain as a major causative factor in infantile autism is gaining favor. It may be necessary to redefine the syndrome when the causal mechanisms are better understood and when primary and secondary symptoms have been distinguished (Rutter M. J Autism Child Schizophr 1978; 8 : 162).

\section{DEGENERATIVE AND FETHBOLIC DISORDERS}

\section{EEG IN RETT'S SYNDROME}

The electroencephalographic (EEG) characteristics of Rett's syndrome were studied in 17 girls between the ages of 1 and 16 yrs at the Sections of Neurophysiology and Pediatric Neurology, Baylor College of Medicine and The Methodist Hospital, Houston, TX. The criteria for the diagnosis of Rett's syndrome included: (1) normal prenatal and perinatal development (2) normal neurological development for the first 7 to 18 mths; (3) cessation of development between 1 and 4 yrs of age and subsequent regression; (4) dementia and autistic features, (5) loss of purposeful use of the hands and development of stereotypical movements; (6) ataxia; (7) acquired microcephaly (8) seizures.

A specific diagnostic EEG pattern was not seen but serial records were characterized by a progressive deterioration: (1) slowing; (2) loss of vertex transients and spindles in sleep; (3) multifocal epileptiform abnormalities; and (4) almost continuous generalized slow spike-and-wave activity. These EEG patterns appeared to correlate with the clinical stages: (1) early onset stagnation; (2) rapid destructive; (3) pseudo-stationary; and (4) late motor deterioration (see Am J Med Genet 1986 (suppl). The EEG of 3 patients was not typical and the average age at onset of their symptoms was later than usual. The authors suggest that the EEG may help to identify variants or atypical cases of Rett's syndrome (Glaze DG et al. Rett's syndrome. Correlation of EEG abnormalities with clinical staging. Arch Neurol $1987 ; 44: 1053-1056$ ).

COMENT. Others have described similar age-related changes in the EEG's of patients with Rett's syndrome. This EEG classification correlated with clinical stages may be useful in diagnosis and prognosis. The imprecise nature of the clinical-EEG correlation, admitted by the authors, may be explained by the nonspecific character and frequency of atypical cases of Rett's syndrome of undetermined etiology.

\section{ATAXIAS}

\section{CEREBELLIAR ATAXIA, OPSOCIONUS, AND NEUROBIASTONA}

A 20 month-old girl with cerebellar ataxia and opsoclonus associated with neuroblastoma is reported from the Pediatric Neurology 